\title{
OCT4 pseudogene 5 upregulates OCT4 expression to promote proliferation by competing with miR-145 in endometrial carcinoma
}

\author{
MINGZHU BAI $^{1 *}$, MU YUAN $^{1,2^{*}}$, HONG LIAO $^{3 *}$, JIAZHOU CHEN $^{1}$, BINYING XIE $^{1}$, DONG YAN $^{1}$, \\ XIAOWEI XI ${ }^{1}$, XIANMING XU $^{1}$, ZHENBO ZHANG $^{1}$ and YOUJI FENG ${ }^{1}$ \\ ${ }^{1}$ Department of Obstetrics and Gynecology, Shanghai First People's Hospital, Shanghai Jiaotong University, Shanghai; \\ ${ }^{2}$ Department of Reproductive Endocrinology, Women's Hospital, School of Medicine, Zhejiang University, \\ Zhejiang; ${ }^{3}$ Clinical and Translational Research Center, Shanghai First Maternity and Infant Hospital, \\ Tongji University School of Medicine, Shanghai, P.R. China
}

Received November 17, 2014; Accepted January 9, 2015

DOI: $10.3892 /$ or.2015.3763

\begin{abstract}
OCT4 plays a critical role in the maintenance of stem cell pluripotency and proliferation, and is overexpressed in multiple human tumors, including endometrial cancer. OCT4 expression can be modulated by miR-145 and the OCT4 pseudogene 5 (OCT4-pg5), which share similar binding sites in the OCT4 3'-untranslated region. The goal of the present study was to evaluate the interaction between miR-145 and OCT4-pg5 on OCT4 expression in endometrial cancer. We assessed OCT4-pg5 expression in 14 benign endometrium and 29 endometrial carcinoma samples. Furthermore, miR-145 mimic transfection was performed to explore its effect on OCT4-pg5 and OCT4 expression, and small interfering RNA (siRNA)-mediated knockdown of OCT4 was conducted to determine whether the effect of OCT4-pg5 on cellular growth was OCT4-dependent. We observed that OCT4-pg5 was abnormally activated in the endometrial carcinomas, and that overexpression of OCT4-pg5 contributed to enhanced cell proliferation and OCT4-PI3K/AKT-cyclin D1 signaling. Moreover, the miR-145 mimic depleted OCT4 expression, whereas elevated OCT4-pg5 restored OCT4 expression and OCT4-PI3K/AKT-cyclin D1 signaling. In conclusion, these data indicate that OCT4-pg5 can act as an RNA sponge to protect OCT4 transcripts from being inhibited by miR-145, providing novel insight into the control of OCT4 expression.
\end{abstract}

Correspondence to: Professor Youji Feng or Dr Zhenbo Zhang, Department of Obstetrics and Gynecology, Shanghai First People's Hospital, Shanghai Jiaotong University, Shanghai 200080, P.R. China E-mail: fengyj4806@sohu.com

E-mail: zhangzhenbozzb@aliyun.com

\section{*Contributed equally}

Key words: endometrial carcinoma, OCT4-pg5, miR-145, OCT4, proliferation

\section{Introduction}

Endometrial carcinoma is the fourth leading cause of cancer-related deaths in women worldwide, with an incidence of approximately $2.26 \%$ of the total newly occurring cases among females in 2008 (1). Surgery, chemotherapy, radiation and hormonal therapy are common types of treatment for patients with endometrial cancer (2). Since each patient may have a different genetic background and pathogenesis, identifying mechanistic differences at the molecular level will benefit the development of patient-specific therapies. Extensive research focusing on endometrial carcinoma has identified several molecules (e.g. PTEN, CA125 and CA199) that are associated with its initiation (3-5); however, its molecular pathogenesis has not been fully elucidated.

OCT4 is a member of the POU domain-containing family of transcription factors, that is primarily expressed in embryonic stem (ES) and primordial germ cells, playing an important role in maintaining pluripotency and self-renewal (6-10). It is also known that OCT4, together with three other reprogramming factors, is essential for generating induced pluripotent stem cells $(11,12)$. Notably, OCT4 expression is closely related to the occurrence, development and metastasis of multiple tumors (13-15), suggesting that OCT4 plays a critical role in carcinogenesis. Previously, we reported that aberrant expression of OCT4 in ovarian cancer was associated with cancer cell anti-apoptotic activity (16). It is known that OCT4 is overexpressed in endometrial carcinoma (17), although little is known concerning the function of OCT4 during endometrial carcinogenesis.

MicroRNAs (miRNAs) are small single-stranded RNAs which can suppress gene expression transcriptionally and post-transcriptionally. The effects of miRNAs are mediated by sequence-specific binding within the 3'-untranslated regions (UTRs) of cognate mRNA targets, typically resulting in the inhibition of translation or mRNA degradation (18). A recent study showed that the tumor-suppressive miRNA, miR-145, directly inhibited the pluripotency factors OCT4, SOX2 and KLF4 in ES cells, and that miR-145 inhibited the transcrip- 
tion of OCT4 by binding to its promoter (19), suggesting that it may play a critical role in OCT4 dysregulation in carcinogenesis (20).

In 2011, Salmena et al proposed the competing endogenous RNA (ceRNA) hypothesis, positing that certain RNAs (i.e. pseudogenes and long non-coding RNAs) regulate biological function by competing for miRNA binding (21). Prior to this hypothesis, pseudogenes were considered to be non-functional genomic sequences (22); however, it has subsequently been shown that many pseudogenes demonstrate biological activity by regulating their homologous genes in cells $(23,24)$. More recent studies indicate that pseudogene transcripts can both regulate tumor oncogenes and suppressor genes by forming small interfering RNAs (siRNA) or reducing the concentration of miRNAs in cells $(17,25,26)$. It has also been directly shown that pseudogenes can function as ceRNAs to regulate other RNA transcripts by competing for shared miRNAs (27). The OCT4 pseudogene 5 (OCT4-pg5) is truncated at the 5'-end and expresses only a partial open reading frame region followed by a 3'UTR. Furthermore, it is the target of miR-145 $(27,28)$. Notably, Suo et al found that OCT4-pg5 was exclusively expressed in cancer tissues but not in normal tissues (15).

The goal of the present study was to determine the expression level of OCT4-pg5 in endometrial cancer, and explore the possibility that it acts as a ceRNA to regulate OCT4 expression, and activate the OCT4-mediated PI3K/AKT-cyclin D1 signaling pathway by competitively binding with miR-145.

\section{Materials and methods}

Reagents. The primary antibodies raised against OCT4 (ab19857), AKT (ab126811), PCNA (ab29), GAPDH (ab8245)and cyclin D1 (ab134175) were all purchased from Abcam (Cambridge Science Park, Cambridge, UK), and the anti-p-AKT (244F9) antibody was purchased from Cell Signaling Technology (Danvers, MA, USA). siRNA specifically targeting OCT4 (1027416) and negative control siRNA were obtained from Qiagen (Dusseldorf, Germany). The 3'UTR of OCT4-pg5 and OCT4 plasmids were constructed by GeneChem (Shanghai, China). The miRNA inhibitor negative control, miR-145 inhibitor (4464084), miRNA mimic negative control, and miR-145 mimic (4464066) were obtained from Ambion (Austin, TX, USA). Lipofectamine 2000, TRIzol reagent, antibiotics and antimycotics (Invitrogen, Grand Island, NY, USA); M-MLV reverse transcriptase (Promega, Madison, WI, USA), Dulbecco's modified Eagle's medium (DMEM), RPMI-1640, fetal bovine serum (FBS; Thermo Scientific, South Logan, UT, USA), SYBR-Green (Takara, Dalian, China) and PCR primers (Shanghai Sangon Biotech, Shanghai, China) were all commercially purchased.

Cell lines and tissue samples. The endometrial cancer cell lines Ishikawa, AN3CA, SPEC-2, HEC-1b, KLE and RL95-2 were maintained by our laboratory. Ishikawa, AN3CA, SPEC-2, HEC-1b and KLE cells were cultured in DMEM with 10\% FBS. RL95-2 cells were cultured in RPMI-1640 medium with $10 \%$ FBS. All cell lines were maintained with $100 \mathrm{IU} / \mathrm{ml}$ penicillin and $100 \mu \mathrm{g} / \mathrm{ml}$ streptomycin in humidified $5 \% \mathrm{CO}_{2}$ at $37^{\circ} \mathrm{C}$. Fourteen samples of fresh human endometrium and 29 cases of cryopreserved endometrial carcinoma were obtained with informed consent from patients in the Shanghai First People's Hospital of Jiaotong University (Shanghai, China). Half of the fresh endometrium samples were in the proliferative phase and the other half were in the secretory phase. All endometrial carcinoma tissues were diagnosed as endometrioid adenocarcinoma. The use of clinical specimens in the present study was approved by the Ethics Committee of Shanghai First People's Hospital of Jiaotong University.

Transient transfection. Transfections were performed using Lipofectamine 2000 following the manufacturer's instructions (Invitrogen). Briefly, the cells were seeded in 60-mm dishes at a density of $1 \times 10^{4}$ cells/well and cultured to $50-60 \%$ confluency. After serum starvation for $24 \mathrm{~h}$, the double-stranded miR-145 mimic, inhibitor, OCT4 siRNA or plasmids were transfected into the cells with Lipofectamine 2000. Twenty four or $48 \mathrm{~h}$ later, the cells were collected for RNA and protein analysis. The sequences of the miR-145 mimic, inhibitor and OCT4 siRNA are listed in Table I.

RNA isolation and real-time quantitative PCR. Total RNA was isolated using TRIzol reagent (Invitrogen) following the manufacturer's recommendations. Complementary DNA was synthesized from $2.5 \mu \mathrm{g}$ of total RNA with random primers and M-MLV reverse transcriptase in a $25-\mu 1$ reaction system. The cDNA template $(2 \mu \mathrm{l})$ was used in a $20-\mu \mathrm{l}$ reaction volume with SYBR-Green for real-time PCR. GAPDH served as an internal normalization control. The PCR primers for OCT4, OCT4-pg5 and GAPDH are listed in Table II. Amplification was carried out for 38 cycles consisting of $15 \mathrm{sec}$ at $95^{\circ} \mathrm{C}$ and $30 \mathrm{sec}$ at $62.4^{\circ} \mathrm{C}$ for OCT4-pg5, and $64^{\circ} \mathrm{C}$ for OCT4, then $30 \mathrm{sec}$ at $72^{\circ} \mathrm{C}$. The $2^{-\Delta \Delta \mathrm{CT}}$ method was used to determine relative gene expression levels, with the mRNA levels normalized to GAPDH expression. Each experiment was repeated in triplicate.

Western blot analysis. Protein was isolated using cell lysis buffer supplemented with a protease and phosphatase inhibitor. Isolated protein $(30 \mu \mathrm{g} /$ lane) was separated on $10 \%$ sodium dodecyl sulfate-polyacrylamide gels, transferred to polyvinylidene fluoride membranes, and then blocked with 5\% skimmed milk for $1 \mathrm{~h}$. Membranes were incubated overnight with the primary antibodies (OCT4, 1:1,000; AKT, 1:1,000; PCNA, 1:1,000; GAPDH, 1:10,000 and cyclin D1 1:10,000). Antibody binding was revealed after incubation with an appropriate secondary antibody coupled to peroxidase by using enhanced chemiluminescence.

Cell proliferation assay. To assess cell proliferation, the cells were stained with sulforhodamine B (SRB). Briefly, the cells were trypsinized and seeded in 96-well plates for experimental assessment $24 \mathrm{~h}$ after transfection. The following day (d 0), 1 plate per day was washed twice with PBS and fixed in $10 \%$ trichloroacetic acid solution at $4^{\circ} \mathrm{C}$. On day 5, all the plates were stained with $0.4 \% \mathrm{SRB}$ for $30 \mathrm{~min}$ at room temperature, and rinsed with $1 \%$ acetic acid 4 times. After lysis with $10 \mathrm{mM}$ Tris, the optical density (OD) of the cell lysates was measured at $490 \mathrm{~nm}$ using a microplate reader (Bio-Rad, Hercules, CA, USA). This experiment was performed in 96-well plates with 6 replicate wells at least 3 times. 
Table I. MicroRNA and OCT4 siRNA sequences.

Name

Sequence

hsa-miR-145 mimic

hsa-miR-145 inhibitor

OCT4 siRNA

GUCCAGUUUUCCCAGGAAUCCCUGGAUUCCUGGGAAAACUGGACUU

AGGGAUUCCUGGGAAAACUGGACCAGUACUUUUGUGUAGUACAA

CAUGUGUAAGCUGCGGCCCTTGGGCCGCAGCUUACACAUGTT

siRNA, small interfering RNA.

Table II. Target gene PCR primer sequences.

\begin{tabular}{llc}
\hline Gene & \multicolumn{1}{c}{ Sequence } & Annealing temperature $\left({ }^{\circ} \mathrm{C}\right)$ \\
\hline OCT4 & & \\
Forward primer & 5'-TCCCTTCGCAAGCCCTCAT-3' & 64 \\
Reverse primer & 5'-TGACGGTGCAGGGCTCCGGGGAGGCCCCATC-3' & 62.4 \\
Oct4-pg5 & & \\
Forward primer & 5'-TTGCTGCAGAAGTGGGTGGAGGAAG-3' \\
Reverse primer & 5'-GTACCAAAATGGGAGCCTGGGGC-3' \\
GAPDH & & 60 \\
Forward primer & 5'-GCACCGTCAAGGCTGAGAAC-3' \\
Reverse primer & 5'-TGGTGAAGACGCCAGTGGA-3' \\
\hline
\end{tabular}

The primers of OCT4 and Oct4-pg5 above were designed by Suo et al (15).

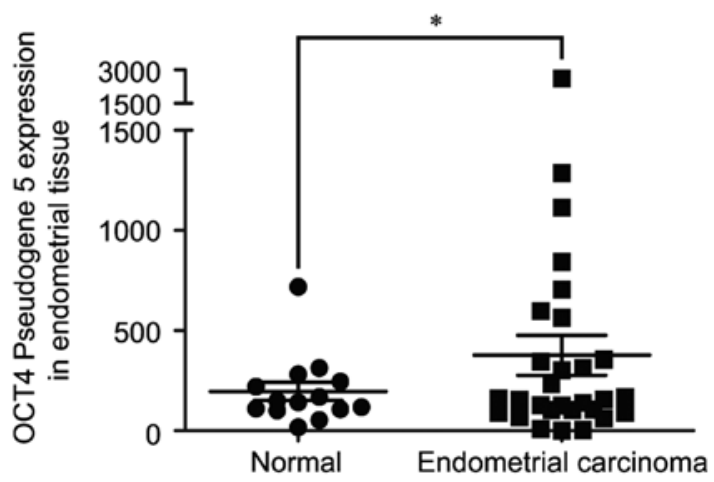

Figure 1. The OCT4-pg5 gene expression profile in normal and cancerous endometrial tissues. Comparison of the OCT4-pg5 mRNA expression level in benign endometrial tissues and the endometrial carcinoma tissues was performed using real-time PCR. " $\mathrm{P}<0.05$ vs. benign endometrial tissues.

Colony formation assay. After the cells were transfected with the plasmids or the miR-145 mimic, they were seeded at $\sim 600$ cells/well in a 6-well plate and cultured for 10 days. Following paraformaldehyde fixation and crystal violet staining, the stained cell colonies were counted. The experiment was carried out in triplicate wells and repeated 3 times.

Statistical analyses. Statistical analysis was performed using SPSS 21.0 (IBM, Armonk, NY, USA). All data are presented as mean \pm standard deviation (SD). Statistical significance of the real-time PCR and cell growth assay was analyzed by the Student's t-test. A P-value $<0.05$ was considered to indicate a statistically significant result.

\section{Results}

OCT4-pg5 is overexpressed in endometrial cancer and is directly targeted by miR-145. To investigate the relative expression level of OCT4-pg5 in endometrial cancer, real-time PCR was performed to assess its mRNA expression level (Fig. 1). OCT4-pg5 mRNA levels were significantly higher in the endometrial cancer samples compared to the level in the normal endometrial tissues $(\mathrm{P}<0.05)$. Further studies revealed that OCT4-pg5 expression was also significantly elevated in the endometrial cancer cell lines AN3CA, Ishikawa and KLE, with an upward trend in HEC-1B cells and no change in SPEC-2 cells, as compared to the expression level in the primary cultured endometrial cells (Fig. 2A). The elevated level of OCT4-pg5 in endometrial cancers suggests that it may play a functional role during carcinogenesis.

It has been previously reported that miR-145 can regulate OCT4 expression by targeting its 3'UTR in various cancer types $(17,29)$. Given that OCT4-pg5 and miR-145 share a binding site within the OCT4 3'UTR (Fig. 3A), it was hypothesized that miR-145 may also target OCT4-pg5. In agreement with this hypothesis, it was observed that treatment with $30 \mathrm{nM}$ with miR-145 mimic in Ishikawa cells resulted in a significant reduction in OCT4-pg5 expression, compared with the negative control group (Fig. 2B). On the other hand, treatment with a miR-145 inhibitor resulted in a potent increase in OCT4-pg5 expression, compared to the negative control (Fig. 2C).

OCT4 is directly targeted by miR-145. In order to confirm previous studies that miR-145 regulates OCT4 

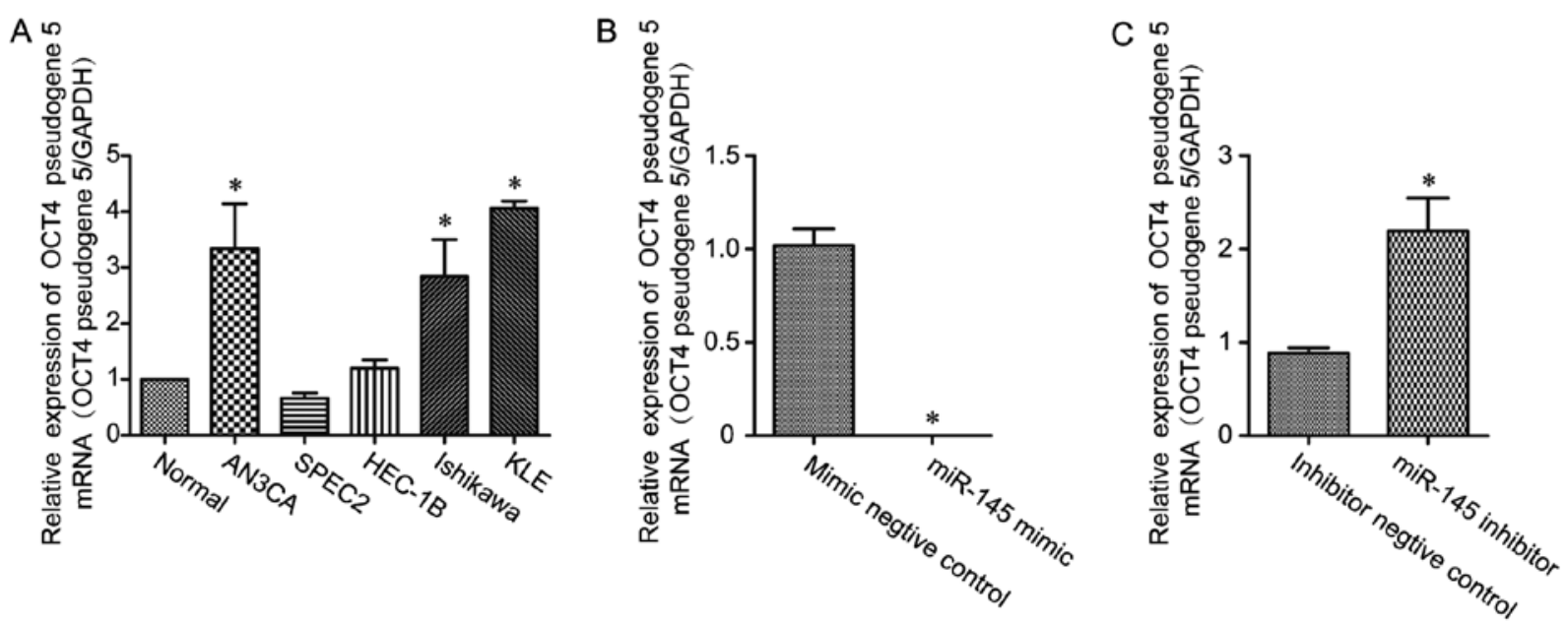

Figure 2. OCT4-pg5 is targeted by miR-145. (A) The relative expression levels of OCT4-pg5 in multiple endometrial carcinoma cell lines. ${ }^{*} \mathrm{P}<0.05$ compared with primary cultured normal endometrial cells. The effects of (B) $30 \mathrm{nM}$ of the miR-145 mimic and (C) $30 \mathrm{nM}$ of the miR-145 inhibitor on OCT4-pg5 expression in Ishikawa cells. ${ }^{*} \mathrm{P}<0.05$ compared with the respective negative control.

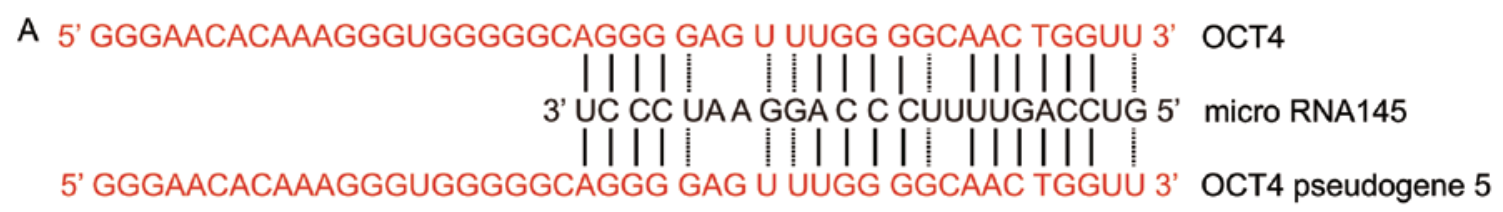

B

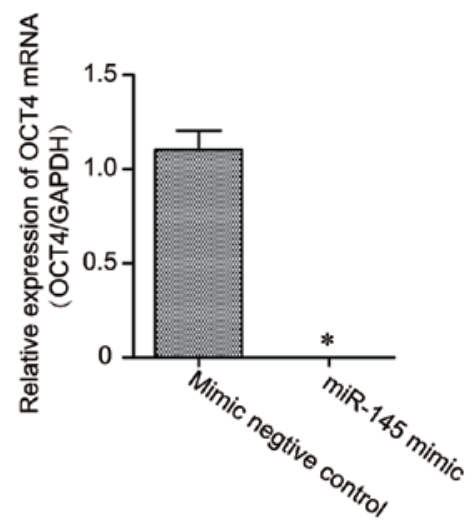

D

$$
\text { OCT4 }
$$

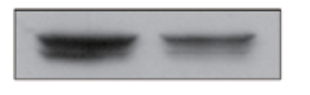

GAPDH

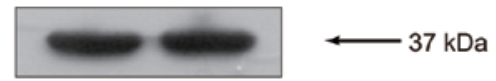

C

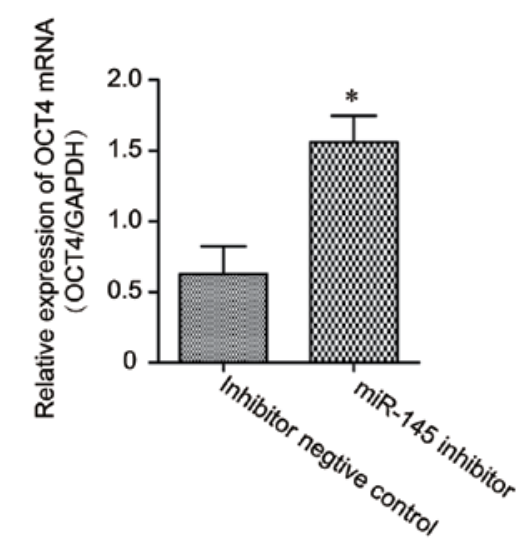

$\mathrm{E}$

OCT4

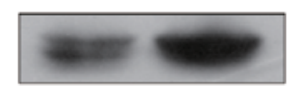

$\longleftarrow 43 \mathrm{kDa}$

GAPDH

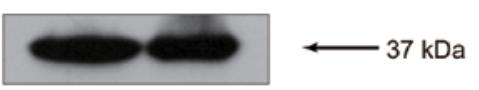

Figure 3. OCT4 is regulated by miR-145. (A) Sequence alignment of miR-145 with the OCT4 and OCT4-pg5 3'UTR. The effect of $30 \mathrm{nM}$ of the miR-145 mimic on (B) OCT4 mRNA and (D) protein expression levels in Ishikawa cells. The effect of $30 \mathrm{nM}$ miR-145 inhibitor on (C) OCT4 mRNA and (E) protein expression levels in Ishikawa cells. ${ }^{~} \mathrm{P}<0.05$ compared with the respective negative control. All western blot analyses are representative.

expression (17,29), OCT4 expression was assessed in the Ishikawa cells treated with $30 \mathrm{nM}$ of the miR-145 mimic. The results showed a marked suppression in the OCT4 mRNA (Fig. 3B) and protein levels (Fig. 3D) in the cells treated with the miR-145 mimic, as compared to the levels in the negative control group. Conversely, treatment with the miR-145 inhibitor was associated with increased OCT4 mRNA (Fig. 3C) and protein expression levels (Fig. 3E), when compared with these levels in the inhibitor negative control. These data suggest that miR-145 regulates OCT4 expression via transcriptional inhibition in Ishikawa cells.

OCT4-pg5 functions as an antagonist against miR-145 binding to OCT4 to promote proliferation. The data presented above indicated that OCT4-pg5 may function as an antagonist against miR-145 binding with the OCT4 promoter in Ishikawa cells. 
A
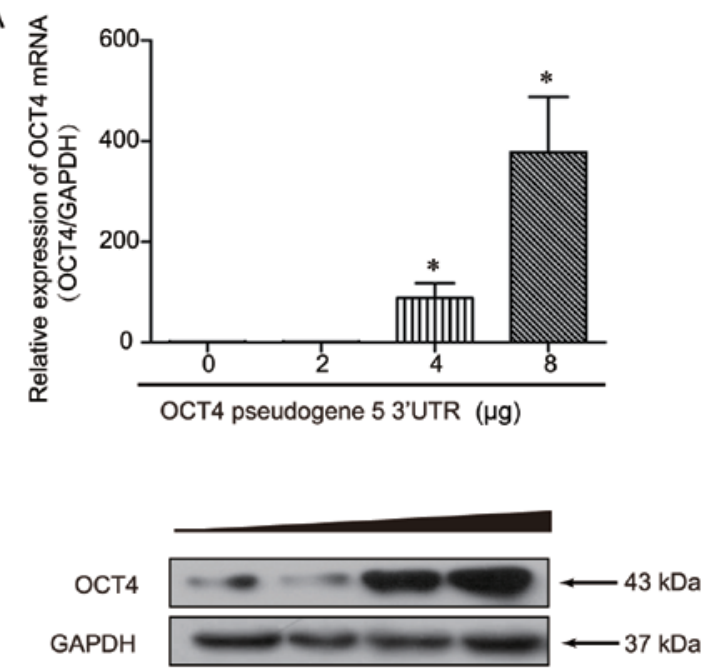

D

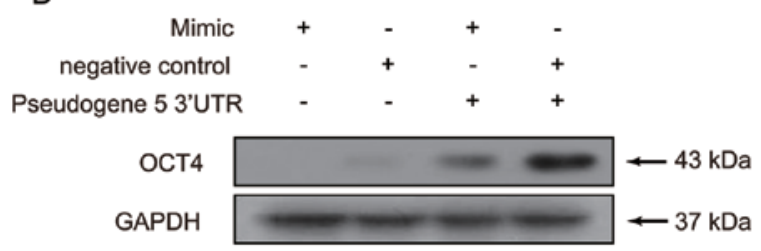

F

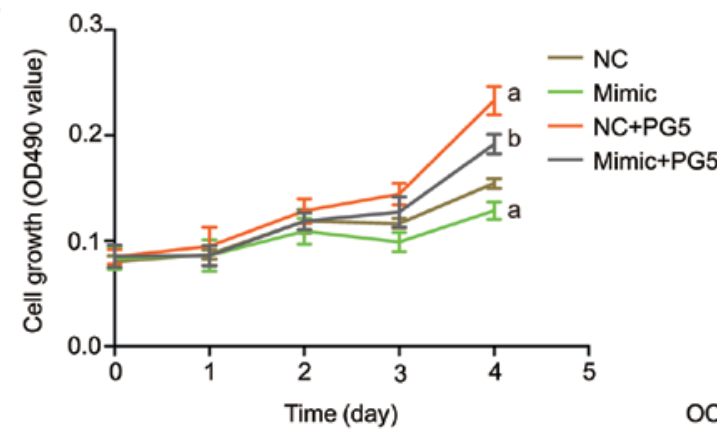

B

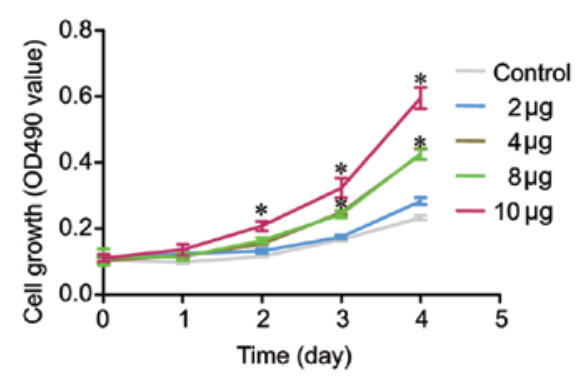

C

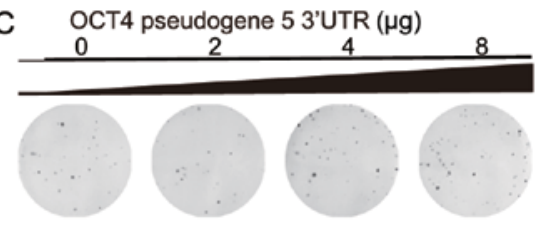

E

OCT4 pseudogene 5 3'UTR $(\mu \mathrm{g})$
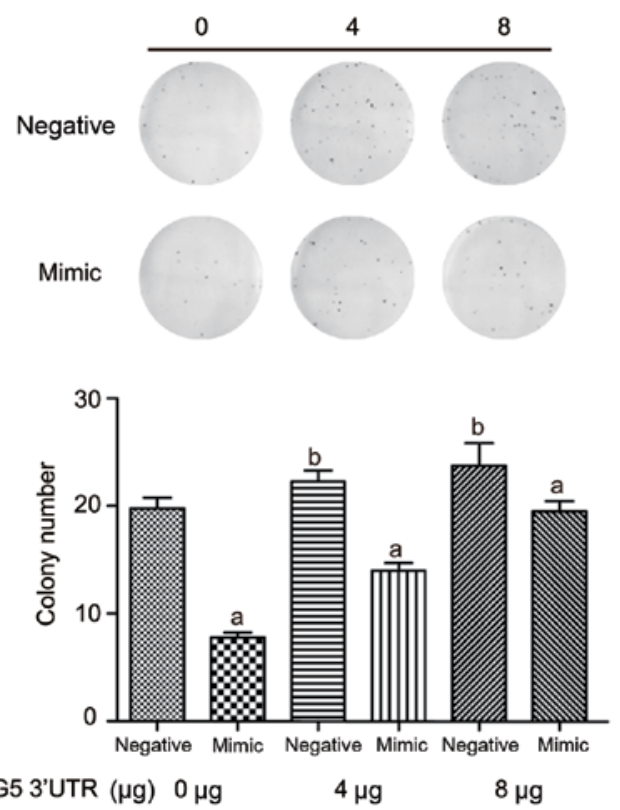

Figure 4. OCT4-pg5 upregulates OCT4 expression by competing for miR-145 in Ishikawa cells. (A) Overexpression of OCT4-pg5 3'UTR elevates OCT4 expression at the mRNA and protein levels. (B) The dose-dependent effect of transfection with the OCT-pg5 3 'UTR on Ishikawa cell proliferation. ${ }^{*} \mathrm{P}<0.05$. (C) Representative images of crystal violet-stained colonies are presented, demonstrating the dose-dependent effect of transfection with OCT-pg5 3'UTR on colony formation in Ishikawa cells. (D) Treatment with $30 \mathrm{nM}$ of the miR-145 mimic attenuates the OCT-pg5-induced expression of OCT4 and (E) has a dose-dependent effect on colony formation. Upper panels show representative images of crystal violet-stained colonies and the lower panel shows quantification of cell colonies between experimental groups: a, compared with each negative control; b, compared with non-overexpressing group. (F) The combined effect of OCT4-pg5 and miR-145 treatment on cell proliferation. All western blot analyses are representative.

To investigate this interaction, overexpression of OCT4-pg5 3'UTR was performed in Ishikawa cells (Fig. 4). It was observed that increasing the expression level of OCT4-pg5 3'UTR markedly elevated the OCT4 mRNA and protein levels in a dose-dependent manner (Fig. 4A). In terms of its biological function, our SRB assay showed that overexpression of the OCT4-pg5 3'UTR significantly accelerated cell proliferation (Fig. 4B) and increased the colony formation rate (Fig. 4C).

Given that miR-145 targets both OCT4 and OCT4-pg5, we investigated whether OCT4-pg5 regulates OCT4 expression by competitive binding with miR-145. As expected, OCT4-pg5-induced OCT4 expression was attenuated by treat- ment with the miR-145 mimic (Fig. 4D), which we attributed to reduced miR-145 mimic binding to the OCT4 3'UTR as a result of increasing miR-145 mimic absorption by elevated OCT4-pg5 3'UTR. We subsequently observed that the exogenous miR-145 mimic also inhibited OCT4-pg5-induced clone formation (Fig. 4E), although a similar proliferative profile was observed (Fig. 4F), suggesting that the miR-145 mimic only partly blocked the role of OCT4-pg5 3'UTR on proliferation. All things considered, these results imply that the increase in OCT4 expression and cell proliferation mediated by OCT4-pg5 overexpression was largely dependent on endogenous miR-145 expression in endometrial cancer. It also 
A

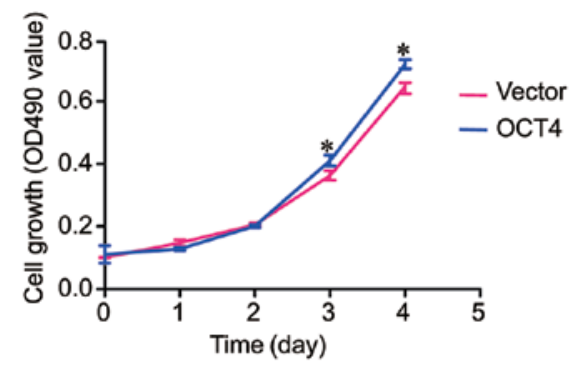

C

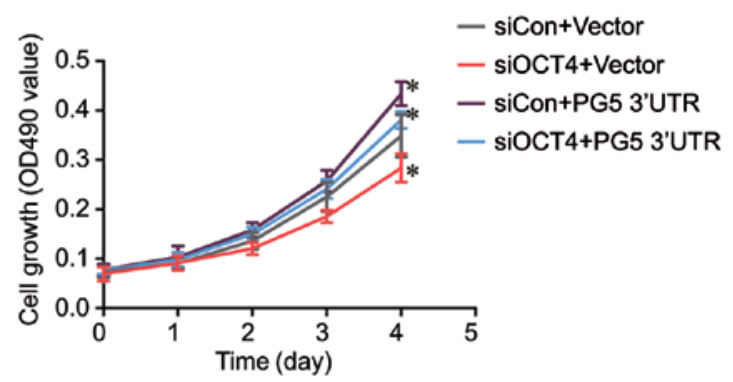

B

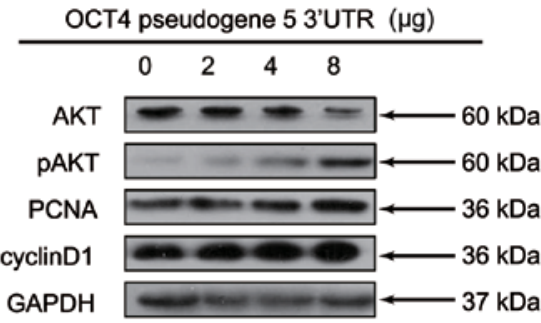

D

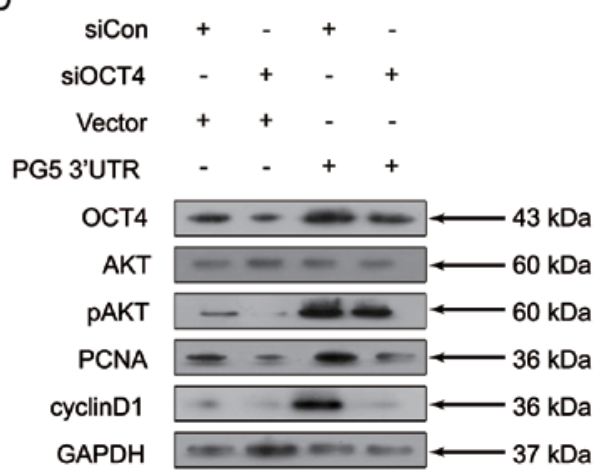

Figure 5. OCT4-pg5-induced Ishikawa cell growth is dependent on the OCT4-PI3K/AKT-cyclin D1 signaling pathway and PCNA. (A) The cell proliferation curve of Ishikawa cells transfected with OCT4 3'UTR. "P<0.05. (B) The dose-dependent effect of OCT4-pg5 overexpression on the protein expression level of AKT, pAKT, PCNA, and cyclin D1. (C) The cell proliferation curve of Ishikawa cells transfected with OCT4-pg5 and OCT4 siRNA ("P<0.05), as well as (D) the effects of these treatment regimens on OCT4, AKT, pAKT, PCNA, and cyclin D1 expression (all western blot analyses are representative).

suggests that OCT4-pg5 functions as a competitive antagonist of miR-145 to protect OCT4 from being repressed by miR-145 in endometrial cancer.

OCT4-pg5-mediated Ishikawa cell proliferation is dependent on OCT4-PI3K/AKT-cyclin DI signaling. To investigate whether OCT4-pg5-induced proliferation is dependent on the OCT4-PI3K/AKT-cyclin D1 signaling pathway in Ishikawa cells, the role of OCT4 on cellular growth and the effect of OCT4-pg5 3'UTR on this signaling pathway were studied. Similar to the effect of OCT4-pg5 overexpression described above (Fig. 4B), increased expression of OCT4 was associated with increased cell proliferation (Fig. 5A). Dissection of the OCT4-PI3K/AKT-cyclin D1 signaling pathway in these cells revealed that OCT4-pg5 3'UTR transfection resulted in increased protein expression levels of p-AKT, PCNA and cyclin D1 (Fig. 5B). To confirm that activation of the PI3K/AKT-cyclin D1 signaling pathway was mediated by OCT4, knockdown of OCT4 or overexpression of OCT4-pg5 3'UTR was performed (Fig. 5C and D). The results from these studies demonstrated that downregulation of $\mathrm{p}$-AKT, PCNA and cyclin D1 by the miR-145 mimic was rescued by overexpression of OCT4-pg5, and their upregulation by OCT4-pg5 was blocked by OCT4 siRNA (Fig. 5D). Similarly, the increased cell proliferation induced by OCT4-pg5 3'UTR was also attenuated by OCT4 siRNA (Fig. 5C).

\section{Discussion}

The molecular mechanisms underlying the development of endometrial carcinoma have not been fully elucidated.
Recently, OCT4 was observed to be aberrantly expressed in endometrial carcinoma, with a resultant association with malignancy $(17,30,31)$. Aberrant OCT4 expression has also been detected in a broad spectrum of other cancers, including non-small cell lung cancer, hepatoma, breast and bladder cancer (32-35). Indeed, it has been suggested that elevated expression of OCT4 contributes to cancer chemotherapy resistance, anti-apoptotic properties, proliferation and invasion (36-39). Previously, we observed that OCT4 is overexpressed in ovarian carcinomas and inhibited FSH-induced apoptosis through enhancing stem cell-like cell expansion and signaling through the OCT4-AKT-survivin pathway (16). All things considered, these facts imply that OCT4 may be an important trigger in carcinogenesis. It has been reported that OCT4 expression is increased in endometrial cancer, that OCT4-induced cell growth was blocked by miR-145 through targeting its 3'UTR, and that OCT4 and miR-145 expression are inversely correlated (17). The goal of the present study was to investigate the factors affecting miR-145 and OCT4 in endometrial carcinogenesis.

OCT4 pseudogenes are reportedly overexpressed in various cancer types (15). In agreement with this notion, we observed markedly elevated OCT4-pg5 expression levels in primary endometrial carcinoma samples and several endometrial cancer cell lines. Indeed, consistent with $\mathrm{Wu}$ et al, we observed that upregulation of OCT4-pg5 was correlated with OCT4 expression (17). Furthermore, exogenous transfection of OCT4-pg5 3'UTR resulted in a dose-dependent increase in OCT4 at the mRNA and protein levels, suggesting that OCT4 is a target of OCT4-pg5, and that this pseudogene is a critical factor affecting OCT4-mediated carcinogenesis. 
Our subsequent experiments were designed to gain a mechanistic insight into the role of OCT4-pg5, with special emphasis on miR-145. It is known that OCT4 can be transcriptionally activated by itself (40), and negatively regulated by miR-145 (19). Indeed, miR-145 is a well-known tumor-suppressor miRNA that is downregulated in numerous types of malignancies, including endometrial cancer $(17,41)$. Poliseno et al previously suggested that OCT4 and its pseudogenes share a similar sequence and have conserved microRNA binding sites (27). In this regard, we confirmed by sequence alignment that there is a conserved miR-145 binding site in the OCT4 and OCT4-pg5 3'UTR. Furthermore, treatment with the miR-145 mimic potently inhibited both OCT4 and OCT4-pg5 expression, whereas its inhibitor sharply increased their expression levels. These data indicate that miR-145 is an important modulator of OCT4-pg5's regulation of OCT4 expression.

All transcribed pseudogenes are theoretically capable of competing with cognate mRNAs for miRNA binding; however, a small percentage of pseudogenes could be processed into endogenous siRNAs to inhibit the expression of cognate genes through RNA interference (42). Ebert et al introduced the concept of 'miRNA sponges' as a means to create lossof-function phenotypes for miRNAs in mammalian cells (43). Similarly, Poliseno et al found that the mammalian pseudogene PTENP1 could function as a ceRNA to regulate PTEN expression by competing for shared miRNAs with PTENP1 3'UTR, with a similar effect found between KRAS and its pseudogene KRAS1P (27). In the context of the present study, Wang et al reported that OCT4-pg4 functions as a natural microRNA sponge to regulate OCT4 expression by competing for miR-145 in hepatocellular carcinoma (44). Here, we observed that the inhibition of OCT4 expression by the miR-145 mimic could be reversed by overexpression of OCT4-pg5 3'UTR, indicating that exogenous OCT4-pg5 3'UTR 'absorbed' more miR-145, thereby preventing it from binding to the OCT4 3'UTR. These data are consistent with the hypothesis that OCT4-pg5 upregulates OCT4 expression by competing against miR-145 in endometrial cancer.

Our subsequent functional studies demonstrated that OCT4-pg5 promoted the cell proliferation and colony-forming ability in endometrial cancer, and that the negative effects of the miR-145 mimic on these parameters could be restored by OCT4-pg5. It was also observed that knockdown of OCT4 in Ishikawa cells resulted in a lower rate of cell proliferation, suggesting an oncogenic role for OCT4 in endometrial carcinogenesis. In our previous studies, the OCT4-PI3K/AKT-survivin signaling pathway was found to be involved in the development of ovarian cancer (16). Similarly, Hu et al showed that OCT4 may maintain the survival of cancer stem cell-like cells in murine 3LL and human MCF7 tumor cell lines partly through Oct4/Tcl1/Akt1 signaling, by inhibiting apoptosis (45). Wang et al also observed that OCT4 can mediate chemoresistance through a potential Oct4-AKT-ABCG2 pathway (36). Here, we demonstrated that overexpression of OCT4-pg5 increased the protein levels of cyclin D1 and PCNA and activated the PI3K/AKT signaling pathway. Nevertheless, OCT4-pg5-induced phosphorylation of AKT and the increase in cyclin D1 and PCNA were blocked by depletion of OCT4, as well as transfection with the miR-145 mimic, suggesting that activating OCT4-PI3K/AKT-cyclin D1 signaling is required for OCT4-pg5 to promote endometrial cancer proliferation by acting as a miR-145 antagonist.

This is the first study to investigate the relationship among OCT4-pg5, miR-145 and OCT4 expression in endometrial carcinogenesis. We observed that the OCT4-pg5 expression level was significantly higher in endometrial carcinoma tissues than in benign endometrial tissues. Our results demonstrate that OCT4-pg5 plays an essential role in miR-145-mediated endometrial carcinoma cell proliferation through regulation of OCT4 expression and the PI3K/AKT-cyclin D1 signaling pathway. These findings provide novel insight into the molecular mechanism of cell proliferation in endometrial carcinoma cells, and may help to reveal novel targets for the treatment of endometrial cancer.

\section{Acknowledgements}

The present study was supported by grants from The National Natural Science Foundation of China (NSFC nos. 81272883, 81202044, 81370074 and 81172478), The Science and Technology Commission of Shanghai Municipality (STCSM no. 12ZR1447600), The Shanghai Municipal Public Health Bureau (no. XYQ2013119), The Shanghai Jiaotong University (no. BXJ201339) and the grant of 'Chenxin plan' from Shanghai Jiaotong University for Z.Z.

\section{References}

1. Jemal A, Bray F, Center MM, Ferlay J, Ward E and Forman D: Global cancer statistics. CA Cancer J Clin 61: 69-90, 2011.

2. NCCN Practice Guidelines in Oncology. Uterine Neoplasms Version 1.2013. http://www.nccn.org/professionals/physician_ gls/f_guidelines.asp\#uterine.

3. Cheng H, Liu P, Zhang F, et al: A genetic mouse model of invasive endometrial cancer driven by concurrent loss of Pten and Lkb1 is highly responsive to mTOR inhibition. Cancer Res 74: 15-23, 2014.

4. Daniilidou K, Frangou-Plemenou M, Grammatikakis J, Grigoriou O, Vitoratos N and Kondi-Pafiti A: Prognostic significance and diagnostic value of PTEN and p53 expression in endometrial carcinoma. A retrospective clinicopathological and immunohistochemical study. J BUON 18: 195-201, 2013.

5. Patsner B and Yim GW: Predictive value of preoperative serum CA-125 levels in patients with uterine cancer: the Asian experience 2000 to 2012. Obstet Gynecol Sci 56: 281-288, 2013.

6. Niwa $\mathrm{H}$ : Molecular mechanism to maintain stem cell renewal of ES cells. Cell Struct Funct 26: 137-148, 2001.

7. Niwa H, Miyazaki J and Smith AG: Quantitative expression of Oct-3/4 defines differentiation, dedifferentiation or self-renewal of ES cells. Nat Genet 24: 372-376, 2000.

8. Pesce M and Scholer HR: Oct-4: control of totipotency and germline determination. Mol Reprod Dev 55: 452-457, 2000.

9. Pesce $M$ and Scholer HR: Oct-4: gatekeeper in the beginnings of mammalian development. Stem Cells 19: 271-278, 2001.

10. Pan GJ, Chang ZY, Schöler HR and Pei D: Stem cell pluripotency and transcription factor Oct4. Cell Res 12: 321-329, 2002.

11. Yu J, Vodyanik MA, Smuga-Otto K, et al: Induced pluripotent stem cell lines derived from human somatic cells. Science 318: 1917-1920, 2007.

12. Takahashi $\mathrm{K}$ and Yamanaka S: Induction of pluripotent stem cells from mouse embryonic and adult fibroblast cultures by defined factors. Cell 126: 663-676, 2006.

13. Wang YD, Cai N, Wu XL, Cao HZ, Xie LL and Zheng PS: OCT4 promotes tumorigenesis and inhibits apoptosis of cervical cancer cells by miR-125b/BAK1 pathway. Cell Death Dis 4: e760, 2013.

14. Monk M and Holding C: Human embryonic genes re-expressed in cancer cells. Oncogene 20: 8085-8091, 2001.

15. Suo G, Han J, Wang X, et al: Oct4 pseudogenes are transcribed in cancers. Biochem Biophys Res Commun 337: 1047-1051, 2005. 
16. Huang Y, Jin H, Liu Y, et al: FSH inhibits ovarian cancer cell apoptosis by up-regulating survivin and down-regulating PDCD6 and DR5. Endocr Relat Cancer 18: 13-26, 2010.

17. Wu Y, Liu S, Xin H, et al: Up-regulation of microRNA-145 promotes differentiation by repressing OCT4 in human endometrial adenocarcinoma cells. Cancer 117: 3989-3998, 2011.

18. Bartel DP: MicroRNAs: target recognition and regulatory functions. Cell 136: 215-233, 2009.

19. Xu N, Papagiannakopoulos T, Pan G, Thomson JA and Kosik KS: MicroRNA-145 regulates OCT4, SOX2, and KLF4 and represses pluripotency in human embryonic stem cells. Cell 137: 647-658, 2009.

20. Hu J, Guo H, Li H, et al: MiR-145 regulates epithelial to mesenchymal transition of breast cancer cells by targeting Oct4. PLoS One 7: e45965 2012.

21. Salmena L, Poliseno L, Tay Y, Kats L and Pandolfi PP: A ceRNA hypothesis: the Rosetta Stone of a hidden RNA language? Cell 146: 353-358, 2011.

22. Petrov DA and Hartl DL: Pseudogene evolution and natural selection for a compact genome. J Hered 91: 221-227, 2000.

23. Hirotsune S, Yoshida N, Chen A, et al: An expressed pseudogene regulates the messenger-RNA stability of its homologous coding gene. Nature 423: 91-96, 2003.

24. Korneev SA, Park JH and O'Shea M: Neuronal expression of neural nitric oxide synthase (nNOS) protein is suppressed by an antisense RNA transcribed from an NOS pseudogene. J Neurosci 19: 7711-7720, 1999.

25. Harrison PM, Zheng D, Zhang Z, Carriero N and Gerstein M: Transcribed processed pseudogenes in the human genome: an intermediate form of expressed retrosequence lacking protein-coding ability. Nucleic Acids Res 33: 2374-2383, 2005.

26. Han YJ, Ma SF, Yourek G, Park YD and Garcia JG: A transcribed pseudogene of MYLK promotes cell proliferation. FASEB J 25 2305-2312, 2011.

27. Poliseno L, Salmena L, Zhang J, Carver B, Haveman WJ and Pandolfi PP: A coding-independent function of gene and pseudogene mRNAs regulates tumour biology. Nature 465 1033-1038, 2010

28. Pain D, Chirn GW, Strassel C and Kemp DM: Multiple retropseudogenes from pluripotent cell-specific gene expression indicates a potential signature for novel gene identification. J Biol Chem 280: 6265-6268, 2005.

29. Zhang S, Wu Y, Feng D, et al: miR-145 inhibits lung adenocarcinoma stem cells proliferation by targeting OCT4 gene. Zhongguo Fei Ai Za Zhi 14: 317-322, 2011 (In Chinese).

30. Mak VC, Siu MK, Wong OG, Chan KK, Ngan HY and Cheung AN: Dysregulated stemness-related genes in gynecological malignancies. Histol Histopathol 27: 1121-1130, 2012.

31. Zhou X, Zhou YP, Huang GR, et al: Expression of the stem cell marker, Nanog, in human endometrial adenocarcinoma. Int J Gynecol Pathol 30: 262-270, 2011.
32. Karoubi G, Gugger M, Schmid R and Dutly A: OCT4 expression in human non-small cell lung cancer: implications for therapeutic intervention. Interact Cardiovasc Thorac Surg 8: 393-397, 2009.

33. Huang PZ, Lu CL, Li BK, et al: OCT4 expression in hepatocellular carcinoma and its clinical significance. Chin J Cancer 29: 111-116, 2010 (In Chinese).

34. Ezeh UI, Turek PJ, Reijo RA and Clark AT: Human embryonic stem cell genes OCT4, NANOG, STELLAR, and GDF3 are expressed in both seminoma and breast carcinoma. Cancer 104 2255-2265, 2005.

35. Xu K, Zhu Z and Zeng F: Expression and significance of Oct4 in bladder cancer. J Huazhong Univ Sci Technolog Med Sci 27 675-677, 2007.

36. Wang XQ, Ongkeko WM, Chen L, et al: Octamer 4 (Oct4) mediates chemotherapeutic drug resistance in liver cancer cells through a potential Oct4-AKT-ATP-binding cassette G2 pathway. Hepatology 52: 528-539, 2010.

37. Zhang Z, Zhu Y, Lai Y, et al: Follicle-stimulating hormone inhibits apoptosis in ovarian cancer cells by regulating the OCT4 stem cell signaling pathway. Int J Oncol 43: 1194-1204, 2013.

38. Li C, Yan Y, Ji W, et al: OCT4 positively regulates survivin expression to promote cancer cell proliferation and leads to poor prognosis in esophageal squamous cell carcinoma. PLoS One 7: e49693, 2012.

39. Dai X, Ge J, Wang X, Qian X, Zhang C and Li X: OCT4 regulates epithelial-mesenchymal transition and its knockdown inhibits colorectal cancer cell migration and invasion. Oncol Rep 29: 155-160, 2013.

40. Pei D: Regulation of pluripotency and reprogramming by transcription factors. J Biol Chem 284: 3365-3369, 2009.

41. Slaby O, Svoboda M, Fabian P, et al: Altered expression of miR-21, miR-31, miR-143 and miR-145 is related to clinicopathologic features of colorectal cancer. Oncology 72: 397-402, 2007.

42. Tam OH, Aravin AA, Stein P, et al: Pseudogene-derived small interfering RNAs regulate gene expression in mouse oocytes. Nature 453: 534-538, 2008.

43. Ebert MS, Neilson JR and Sharp PA: MicroRNA sponges: competitive inhibitors of small RNAs in mammalian cells. Nat Methods 4: 721-726, 2007.

44. Wang L, Guo ZY, Zhang R, Xin B, Chen R, Zhao J, Wang T, Wen WH, Jia LT, Yao LB and Yang AG: Pseudogene OCT4-pg4 functions as a natural micro RNA sponge to regulate OCT4 expression by competing for miR-145 in hepatocellular carcinoma. Carcinogenesis 34: 1773-1781, 2013.

45. Hu T, Liu S, Breiter DR, Wang F, Tang Y and Sun S: Octamer 4 small interfering RNA results in cancer stem cell-like cell apoptosis. Cancer Res 68: 6533-6540, 2008. 